\title{
Towards targeted combinatorial therapy design for the treatment of castration-resistant prostate cancer
}

\author{
Osama A. Arshad \\ Center for Bioinformatics and \\ Genomics Systems Engineering \\ Texas A\&M University \\ College Station, TX 77843 \\ oarshad@tamu.edu
}

\author{
Aniruddha Datta \\ Center for Bioinformatics and \\ Genomics Systems Engineering \\ Texas A\&M University \\ College Station, TX 77843 \\ datta@ece.tamu.edu
}

\begin{abstract}
Prostate cancer is one of the most prevalent cancers in males in the United States and amongst the leading causes of cancer related deaths. A particularly virulent form of this disease is castrationresistant prostate cancer (CRPC), where patients no longer respond to medical or surgical castration. CRPC is a complex, multifaceted and heterogeneous malady with limited standard treatment options. The growth and progression of prostate cancer is a complicated process that involves multiple pathways. The signaling network comprising the integral constituents of the signature pathways involved in the development and progression of prostate cancer is modeled as a combinatorial circuit. The failures in the gene regulatory network that lead to cancer are abstracted as faults in the equivalent circuit and the Boolean circuit model is then used to design therapies tailored to counteract the effect of each molecular abnormality and to propose potentially efficacious combinatorial therapy regimens. Furthermore, stochastic computational modeling is utilized to identify potentially vulnerable components in the network that
\end{abstract}

may serve as viable candidates for drug development. The results presented herein can aid in the design of scientifically wellgrounded targeted therapies that can be employed for the treatment of prostate cancer patients.

\section{Categories and Subject Descriptors}

I.6.3 [Simulation and Modeling]: Applications; J.3 [Life and Medical Sciences]: Biology and Genetics.

\section{General Terms}

Algorithms

\section{Keywords}

Prostate cancer, gene regulatory networks, Boolean modeling, combination therapy
Permission to make digital or hard copies of all or part of this work for personal or classroom use is granted without fee provided that copies are not made or distributed for profit or commercial advantage and that copies bear this notice and the full citation on the first page. Copyrights for components of this work owned by others than the author(s) must be honored. Abstracting with credit is permitted. To copy otherwise, or republish, to post on servers or to redistribute to lists, requires prior specific permission and/or a fee. Request permissions from Permissions@acm.org.

BCB '16, October 2-5, 2016, Seattle, WA, USA

Copyright is held by the owner/author(s). Publication rights licensed to ACM.

Copyright 2016 ACM 978-1-4503-4225-4/16/10_..\$15.00.

http://dx.doi.org/10.1145/2975167.2985671 